\title{
Engineering thermosensitive liposome-nanoparticle hybrids loaded with doxorubicin for heat-triggered drug release
}

\author{
Zahraa Al-Ahmadya, [b], Neus Lozano [a], [b], Kuo-Ching Mei [b], [c], Wafa' T. \\ Al-Jamal [b], [d], Kostas Kostarelos [a], [b], \\ [a] Nanomedicine Lab, Faculty of Biology, Medicine \& Health, University of Manchester, AV Hill Building, \\ Manchester M13 9PT, United Kingdom \\ [b] UCL School of Pharmacy, University College London, London WC1N 1AX, United Kingdom \\ [c] Institute of Pharmaceutical Science, King's College London, 150 Stamford Street, London SE1 9NH, United \\ Kingdom \\ [d] University of East Anglia, Norwich Research Park, Norwich NR4 7TJ, United Kingdom
}

\begin{abstract}
The engineering of responsive multifunctional delivery systems that combine therapeutic and diagnostic (theranostic) capabilities holds great promise and interest. We describe the design of thermosensitive liposome-nanoparticle (NP) hybrids that can modulate drug release in response to external heating stimulus. These hybrid systems were successfully engineered by the incorporation of gold, silver, and iron oxide NPs into the lipid bilayer of lysolipid-containing thermosensitive liposomes (LTSL). Structural characterization of LTSL-NP hybrids using cryo-EM and AFM revealed the incorporation of metallic NPs into the lipid membranes without compromising doxorubicin loading and retention capability. The presence of metallic NPs in the lipid bilayer reinforced bilayer retention and offered a nanoparticle concentration-dependent modulation of drug release in response to external heating. In conclusion, LTSL-NP hybrids represent a promising versatile platform based on LTSL liposomes that could further utilize the properties of the embedded NPs for multifunctional theranostic applications.
\end{abstract}

\section{Graphical abstract}
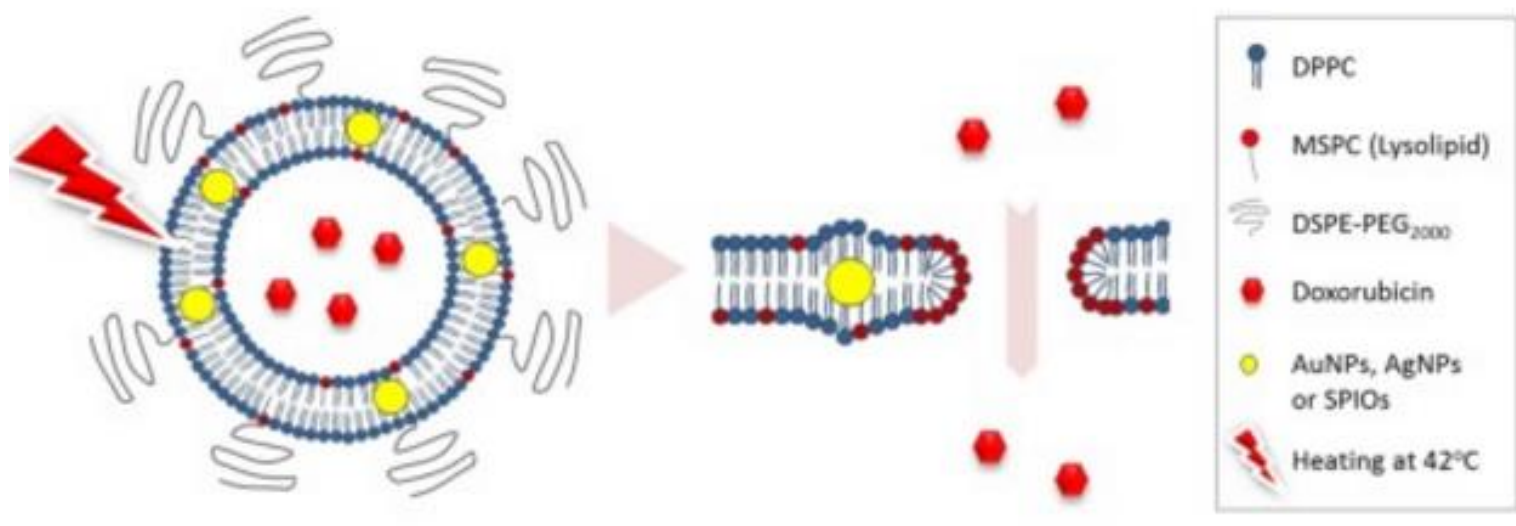


\section{Keywords}

Liposomes; Nanoparticles; Doxorubicin; Nanomedicine

\section{Introduction}

Liposomes are self-assembled phospholipid vesicles that have been clinically approved as a nanoscale delivery system for various therapeutic applications (Allen and Cullis, 2013 and Barenholz, 2012). Liposomes can effectively entrap both hydrophilic (Lozano et al., 2015 and Li et al., 1998) and hydrophobic compounds (Chen et al., 2010, Koudelka and Turánek, 2012 and Lozano et al., 2015) in their aqueous core or the lipid bilayer, respectively. Liposomes are biocompatible, biodegradable and can effectively modify the pharmacokinetic profile of their loaded drugs (Sawant and Torchilin, 2012). Stealth liposomes can passively accumulate at the tumor site as they leak through malformed blood vessels while avoiding healthy tissues and organs (Gabizon et al., 1994 and Gabizon and Martin, 1997). Next-generation liposomes that can trigger-release their drug content in response to external stimuli have been developed to improve drug bioavailability and overall therapeutic efficacy (Al-Ahmady et al., 2014 and Al-Ahmady et al., 2015). The most clinically advanced such vesicle system is commercially known as ThermoDox ${ }^{\circledR}$, a lysolipid-containing temperature-responsive liposome (LTSL) that is currently in clinical trials for treatment of solid tumors in combination with mild hyperthermia (HT) (Al-Ahmady and Kostarelos, 2016).

Over the last few years, advances in nanotechnology have dramatically increased interest in developing metallic nanoparticles (NPs) for a wide range of applications (Grzelczak and LizMarzan, 2013, Hormeno et al., 2014, Johannsen et al., 2010, Liu et al., 2012, Maestro et al., 2014, Visaria et al., 2006 and Wong and Liu, 2010). The nanoscale size of metallic NPs such as gold (AuNPs), silver (AgNPs), superparamagnetic iron oxides (SPIO) and their novel optical and magnetic properties enable their use for both imaging and therapeutic purposes. For example, the enhanced surface plasmon resonance of AuNPs makes them particularly attractive for imaging and photo-thermal applications (Maestro et al., 2014). The absorption of light at certain wavelengths causes the oscillation of surface electrons and subsequent local heat generation, that can be controlled by the intensity of the laser beam irradiation, duration of NP exposure and the concentration of AuNPs (Alkilany et al., 2012).

Similarly, SPIO NPs have been used extensively as a contrast agent for magnetic resonance imaging (MRI). The magnetic properties of SPIO NPs can also be exploited for the generation of magnetically-mediated hyperthermia (Chanda et al., 2010). The exposure of SPIO NPs to an alternating magnetic field can generate high local heating by Neel fluctuations which have showed potential in cancer therapy (Jordan et al., 2006 and Maier-Hauff et al., 2007). NP-induced hyperthermia has the potential to overcome some of the limitations of conventional heating techniques when treating deep or non-accessible tumors (Preiss and Bothun, 2011). However, the inherent hydrophobic surface character of metallic NPs and their limited colloidal stability can significantly affect their biological performance.

We and others have previously shown the possibility of using liposome technology as a delivery platform that could allow the incorporation of metallic NPs (Al-Jamal et al., 2008, Jain et al., 2003 and Lozano et al., 2012). Liposomal incorporation of metallic NPs has proved to be an effective way to increase nanoparticle stability and biocompatibility in biological 
conditions (Lozano et al., 2012 and Preiss and Bothun, 2011). This can be achieved by either the incorporation of hydrophobic NPs within the lipid membrane or encapsulation of the hydrophilic NPs in the liposomal aqueous core (Tai et al., 2009). Alternatively, metallic NPs can be functionalized onto the liposomal surface by either physical or chemical conjugation (Chithrani et al., 2010 and Pornpattananangkul et al., 2010). To date several examples of liposome-NP hybrids have been designed for diagnostic (Soga et al., 2010), or simply for colloidal stabilization (Kojima et al., 2008 and Lozano et al., 2012) purposes, with some interesting examples of smart hybrid systems that can trigger release in response to external stimuli. Such cases of thermosensitive liposome-NP hybrids were designed to utilize the photonic and electromagnetic properties of NPs for local heat generation and triggered content release (Paasonen et al., 2010, Tai et al., 2009 and Wu, 2008). As a proof of concept, several studies have demonstrated the release of encapsulated dye molecules from such hybrid systems (Bealle et al., 2012, Chen et al., 2010 and Paasonen et al., 2010). However, only a few studies have reported the potential of combining thermosensitive liposome-NP hybrids with therapeutic agents for combinatory therapeutic and diagnostic applications (Tian et al., 2011).

The aim of the present work was to engineer and characterize doxorubicin loadedthermosensitive, liposome-NP hybrids as multimodal smart systems to control drug release. Drug (doxorubicin) loaded, LTSL were chosen for this study as the most advanced TSL that are currently in clinical trials (Clesion.com, 2016 and Landon et al., 2011). The incorporation of three different types of metallic NPs into LTSL was studied and the capacity of the hybrid systems to modulate drug release was investigated.

\section{Materials and methods}

\subsection{Materials}

1,2-Dipalmitoyl-sn-glycero-3-phosphocholine (DPPC), 1-stearoyl-2-hydroxy-sn-glycero-3phosphocholine (MSPC), 1,2-distearoyl-sn-glycero-3-phosphoethanolamine- $\mathrm{N}$ [methoxy(polyethylene glycol)-2000] (DSPE-PEG2000) were kind gifts from Lipoid GmbH (Ludwigshafen, Germany). Ammonium sulfate, sodium hydroxide, chloroform and methanol were purchased from Fisher Scientific. 1,6-Diphenyl-I,3,5-hexatriene (DPH) was purchased from Invitrogen. Diethyl ether, doxorubicin hydrochloride (DOX), 4-(2-hydroxyethyl) piperazine-1-ethanesulfonic acid (HEPES), sodium chloride ( $\mathrm{NaCl}$ ), Sephadex ${ }^{\circledR} \mathrm{G}-50$, oleic acid-functionalized magnetic SPIO NPs solution $5 \mathrm{mg} / \mathrm{ml}$ in toluene, octanethiolfunctionalized AuNPs $2 \%$ solution in toluene, decanethiol-functionalized AgNPs $0.1 \%(\mathrm{w} / \mathrm{v})$ in hexane, 8-anilino-1-naphthalenesulfonic acid (ANS), and tetrahydrofuran were purchased from Sigma.

\subsection{Preparation and characterization of liposomes and liposome-NP hybrids}

Lysolipid containing temperature-sensitive liposomes (LTSL) composed of DPPC:MSPC:DSPEPEG2000 86:10:4 (molar ratio) were prepared using the reverse phase evaporation method [RP] and the lipid film-reverse phase evaporation method [F-RP] (Fig. 1A). For the RP method the lipids dissolved in chloroform/methanol mixture were mixed in $25 \mathrm{ml}$ round bottom flask and then $6 \mathrm{ml}$ of chloroform/diethylether $(1: 1, \mathrm{v} / \mathrm{v})$ and $1.5 \mathrm{ml}$ of $240 \mathrm{mM}$ ammonium sulfate buffer ( $\mathrm{pH} 5.4$ ) were added at 1:4 v/v (aqueous/organic). In order to 
form w/o emulsion, the mixture was sonicated for $15 \mathrm{~min}$ in a bath sonicator at $40{ }^{\circ} \mathrm{C}$. Organic solvents were then evaporated using rotary evaporator (BÜCHI, Switzerland) at 460 mbar and $40{ }^{\circ} \mathrm{C}$ for $2 \mathrm{~h}$ resulting in the formation of a gel-like phase followed by aqueous phase formation. Large unilamellar liposomes (LUVs) formed by this process were then reduced in size by $30 \mathrm{~min}$ sonication at $60^{\circ} \mathrm{C}$ using a bath sonicator.

Reverse Phase Evaporation [RP]

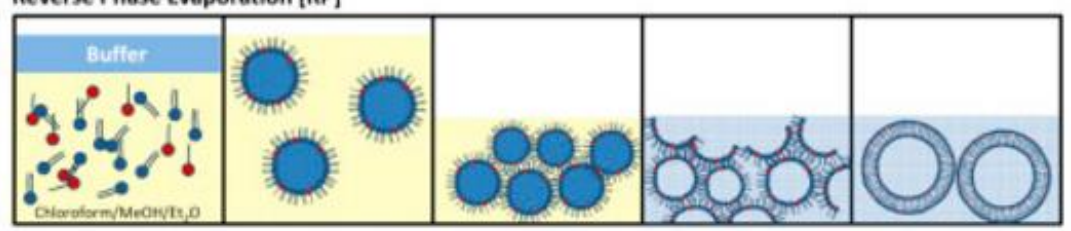

Film - Reverse Phase Evaporation [F-RP]

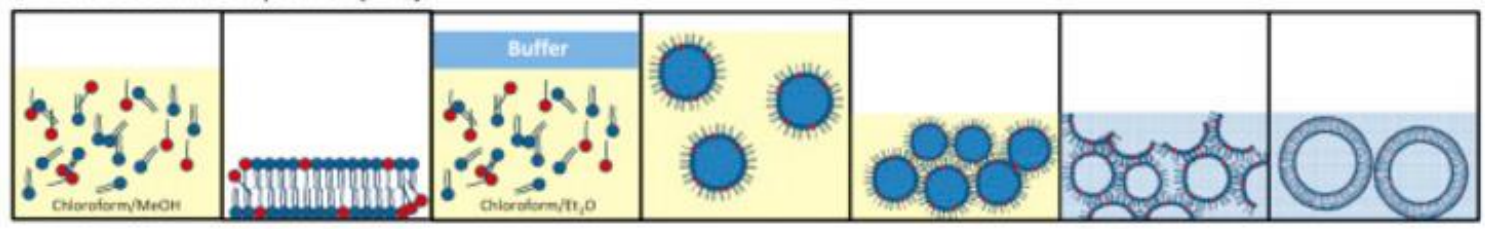

B

\begin{tabular}{|c|c|c|c|c|}
\hline System* & Hydrodynamic diameter" (nm) & $P D I^{*}$ & 6-Potential" (mV) & $E E^{b} \%$ \\
\hline$L T S L[R P]$ & $68.8 \pm 0.5$ & $0.253 \pm 0.011$ & $-24.3 \pm 1.1$ & 86 \\
\hline LTSL $\mid F-R P]$ & $73.0 \pm 0.6$ & $0.304 \neq 0.032$ & $-18.8=0.1$ & 66 \\
\hline
\end{tabular}

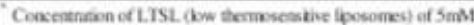

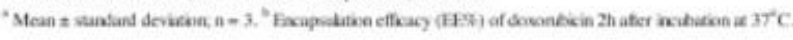

c $i$
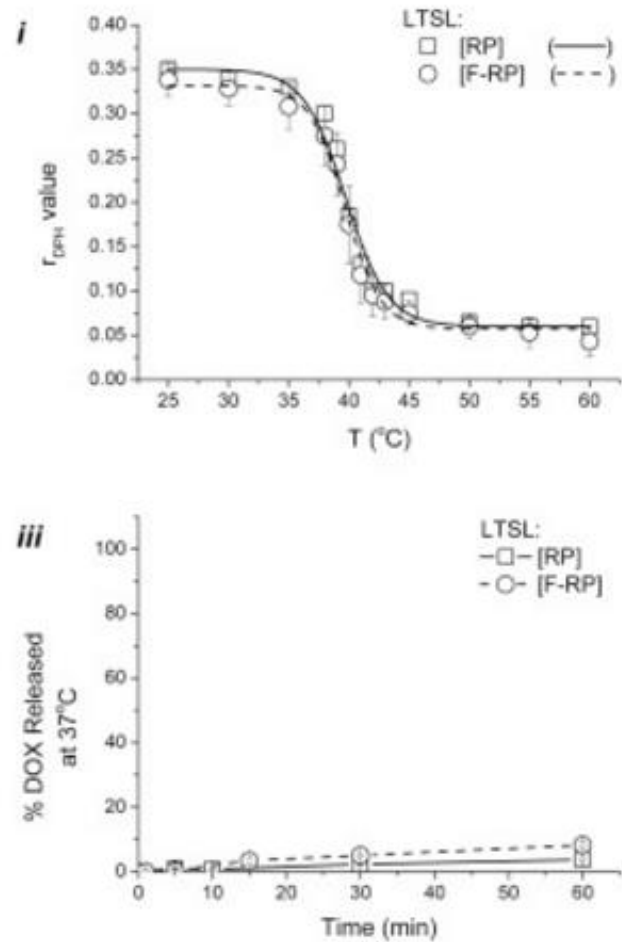

ii $\quad 0227 \ldots$ LTSL
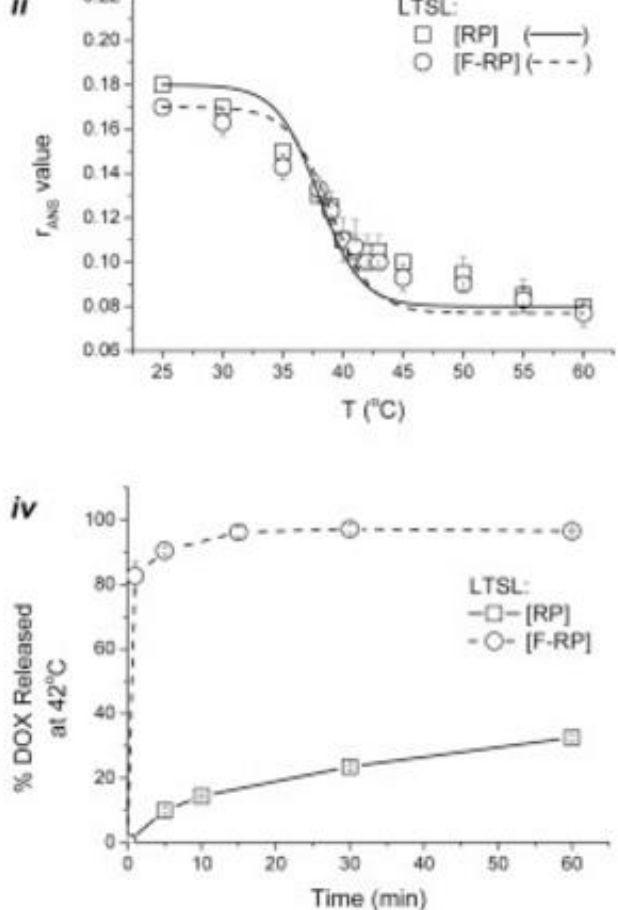

Fig. 1.

(A) Schematic of the protocols used for LTSL preparation: reverse phase evaporation [RP] and film-reverse phase evaporation [F-RP]. (B) Colloidal stability summary and doxorubicin encapsulation efficiency (EE\%) of the LTSL prepared by [RP] and [F-RP]. Data obtained by Nanosizer ZS and fluorescence spectrophotometer. (C) Effect of LTSL preparation protocol: fluorescence anisotropy values of (i) DPH and (ii) ANS and\% doxorubicin released at (iii) $37{ }^{\circ} \mathrm{C}$ and (iv) $42{ }^{\circ} \mathrm{C}$ using the [RP] (squares and solid lines) and [F-RP] (circles and dashed lines) protocols. 
To prepare the liposomes by F-RP method, the same procedure described above was applied with the exception that a lipid film was first prepared by evaporating chloroform:methanol from the lipid mixture. This then followed by re-dissolving the lipid film in the chloroform/diethylether/ammonium sulfate mixture as described earlier.

For the preparation of liposome-NP hybrids, F-RP method was applied. First, the exact amount of hydrophobic NPs dispersion ( $5 \mu \mathrm{g}$ or $10 \mu \mathrm{g}$ ) was added to $25 \mathrm{ml}$ round bottom flask. The organic solvent in which the NPs were dispersed was removed using rotary evaporator at $6 \mathrm{mbar}$ and $40^{\circ} \mathrm{C}$ for $1 \mathrm{~h}$. The lipids mixture of DPPC:MSPC:DSPE-PEG2000 86:10:4 (molar ratio) dissolved in chloroform/methanol (4:1) were then added and the organic solvents were evaporated to form the lipid film containing the NPs. The resulting lipid film was then re-dissolved the lipid film in $6 \mathrm{ml}$ of chloroform/diethylether (1:1). Following this step $1.5 \mathrm{ml}$ of $240 \mathrm{mM}$ ammonium sulfate buffer ( $\mathrm{pH} 5.4)$ was added and the mixture was sonicated for $15 \mathrm{~min}$ in a bath sonicator at $40{ }^{\circ} \mathrm{C}$. Organic solvents were then evaporated using rotary evaporator at $460 \mathrm{mbar}$ and $40^{\circ} \mathrm{C}$ for $2 \mathrm{~h}$ and the size of LUVs of LTSL-Np hybrids was then reduced by bath sonication as described earlier.

Liposome size and surface charge were measured by using Zetasizer Nano ZS (Malvern, Instruments, UK). For size measurement samples were diluted with HEPES buffer saline (HBS) composed of $20 \mathrm{mM}$ HEPES, $150 \mathrm{mM} \mathrm{NaCl}$, pH 7.4 and measured in $1 \mathrm{ml}$ cuvettes. Zeta potential was measured in disposable Zetasizer cuvettes and sample dilution was performed with distilled water.

\subsection{Cryo-electron microscopy (Cryo-EM)}

Visualization of samples with Cryo-EM was performed to study the morphology of LTSL-NPS hybrids and to confirm the incorporation of metallic NPs. Sample preparation was carried out in a temperature- and humidity-controlled chamber using a fully automated (PCcontrolled) vitrification robot (Vitrobot). $46 \mathrm{~A}$ specimen grid was dipped into a suspension and withdrawn, and excess liquid was blotted away. Thin films were formed between the bars of the grids. To vitrify these thin films, the grid was shot into melting ethane. The grids with vitrified thin films were analyzed in a CM-12 transmission microscope (Philips, Eindhoven, The Netherlands) at $170{ }^{\circ} \mathrm{C}$ using a Gatan-626 cryo-specimen holder and cryotransfer system (Gatan, Warrendale, PA). The vitrified films were studied at $120 \mathrm{kV}$ and at standard low-dose conditions.

\subsection{Atomic force microscopy (AFM)}

Sample analysis with AFM was then performed to study the effect of NPs incorporation on the bilayer thickness. $20 \mu \mathrm{l}$ of liposome dispersion in a concentration of $5 \mathrm{mM}$ was deposited on the surface of a newly cleaved mica sheet (Agar Scientific, Essex, UK) and was allowed to adsorb for $1 \mathrm{~min}$. Unbound liposomes were washed out by filtered distilled water and then dried by dust-free compress air. Images were taken in tapping-mode using a Multimode AFM, E-type scanner, Nanoscope IV controller with Nanoscope 5.31r1 control software (Bruker Surface Nano, Cambridge, UK). The tip was a silicon tapping tip with $10 \mathrm{~nm}$ curvature radius (NSG01, NTI-Eutope, Apdldoorn, The Netherlands). Typical resonant frequency of the tapping-mode silicon cantilever of $150 \mathrm{kHz}$ with a force constant of 5.5 
$\mathrm{N} / \mathrm{m}$ was used. A scan rate of $1 \mathrm{~Hz}$ was used to scan a $5 \mu \mathrm{m} 2$ of the mica surface with a resolution of $512 \times 512$ pixels. WSxM 5.0 software was used to process, filter and flatten the images.

\subsection{Differential scanning calorimetry (DSC)}

Phase transition temperature (Tt), of LTSL Liposomes and the three types of LTSL-NP hybrids were studied with DSC. This method is use to study the change in bilayer structure from solid gel-phase into liquid crystalline-phase as the temperature increased. Liposomes were prepared as LUVs by F-RP method. Samples were first prepared at the concentration of 15 $\mathrm{mM}$ and then concentrated 5 times by centrifugation at 13,000 rpm for $30 \mathrm{~min}$ using 0.22 $\mu \mathrm{m}$ Spin-X Centrifuge Tube Filters (Sigma-Aldrich, UK). $20 \mu \mathrm{l}$ of the concentrated liposome dispersion were then transferred and sealed into the hermetic Tzero ${ }^{\mathrm{TM}}$ DSC pan and analyzed by Q2000 DSC (TA Instruments, USA) at $1{ }^{\circ} \mathrm{C}$ per min from $30^{\circ} \mathrm{C}$ to $60^{\circ} \mathrm{C}$.

\subsection{Fluorescence anisotropy measurements}

Fluorescence anisotropy experiments were performed to study the effect of metallic NPs incorporation on the liposomes membrane fluidity. A hydrophobic probe (DPH) and hydrophilic probe (ANS) were used to monitor the changes in the bilayer fluidity at the hydrophobic region of the lipid membrane and near the liposomes surface, respectively. Experiments were performed as previously described (Al-Ahmady et al., 2012). Briefly, liposomes were further diluted to $0.025 \mathrm{mM}$ and divided into two $4 \mathrm{ml}$ aliquots. DPH solution in tetrahydrofuran $(0.8 \mathrm{mM}, 2.5 \mu \mathrm{l})$ or an aqueous ANS solution $(10 \mathrm{mM}, 4 \mu \mathrm{l})$ was mixed with the liposomes at 500:1 lipid/DPH or 25:1 lipid/ANS. To allow the probes to be incorporated, the samples were shaken at room temperature for $2 \mathrm{~h}$ and then left overnight before starting measurements. Fluorescence polarization was then measured by a LS-50 B spectrofluorometer (Perkin Elmer) equipped with automated polarizer and thermostatic cell holder connected to a water bath to control the sample temperature. For the DPH experiment, the anisotropy measurements were carried out at an excitation wavelength of $395 \mathrm{~nm}$ (slit of $10 \mathrm{~nm}$ ) and emission wavelength of $476 \mathrm{~nm}$ (slit of $5 \mathrm{~nm}$ ). Measurements were started at $25^{\circ} \mathrm{C}$, and then the temperature was increased gradually up to $60^{\circ} \mathrm{C}$. Boltzmann curve fitting of the experimental values were performed using Origin software. The samples were equilibrated for at least 6 min after each temperature change. Fluorescence anisotropy was then measured automatically by the spectrofluorometer based on Eq. (1);

$r=\frac{I_{v}-G l v_{H}}{I v_{v}+2 G l v_{H}}$

where $r$ is the fluorescence anisotropy and IVV and IVH are the emission intensity excited with vertically polarized light and measured with emission polarizer oriented in a parallel or perpendicular direction to the plane of excitation, respectively. $G$ is an instrument specific factor calculated to correct the instrument polarization, which is equal to $\mathrm{IHV} / \mathrm{IHH}$, and obtained by measuring the vertically and horizontally polarized emission intensities after excitation with horizontally polarized light. 


\subsection{Doxorubicin loading using $\mathrm{pH}$-gradient method}

Remote doxorubicin loading into pre-formed liposomes was performed using $\mathrm{pH}$ gradient method. Ammonium sulfate buffer $240 \mathrm{mM}$ (pH 5.4) was used for the liposomes preparation as mentioned earlier then external buffer exchange was performed by gel filtration through Sephadex ${ }^{\circledR}$ G-50 column equilibrated with HBS (pH 7.4). Doxorubicin hydrochloride $(5 \mathrm{mg} / \mathrm{ml}$ ) was added to the liposome suspensions at 20:1 lipid/doxorubicin mass ratio (i.e. $0.187 \mathrm{mg}$ of doxorubicin for $5 \mathrm{mM}$ liposomes). This was then followed by $2 \mathrm{~h}$ incubation at $37^{\circ} \mathrm{C}$.

After incubation liposomes were passed again through Sephadex ${ }^{\circledR}$ G-50 column to remove any free doxorubicin. Encapsulation efficiency (\% EE) was calculated by comparing the total fluorescence intensity of doxorubicin post and pre gel filtration, diluted to the same final lipid concentration using Eq. (2);

$\% E E=I(t)$ post column/I(t) pre column $* 100$

where, $\mathrm{I}(\mathrm{t})$ is the total fluorescence intensity of the liposome suspension after adding $2 \mu \mathrm{l}$ Triton X-100 (10\% in HBS, pH 7.4).

\subsection{Doxorubicin release studies}

Doxorubicin release was measured by taking advantage of the fluorescence quenching process. When doxorubicin is encapsulated inside the liposomes, its concentrations is very high resulting in self-quenching of the fluorescence signal. When the ambient temperature exceeded the liposome Tt, doxorubicin was released from the liposomes and its concentration is diluted resulting in increase in fluorescence intensity, which is used to monitor the release process.

Doxorubicin release studies were performed in HBS. Samples were incubated at $37^{\circ} \mathrm{C}$ and $42{ }^{\circ} \mathrm{C}$ with a shaking water bath (Grant, U.K.). $20 \mu$ S Samples were then measured at different time points at 480/595 nm excitation and emission wavelengths (slit 15/20 nm) using PerkinElmer Luminescence Fluorimeter (LS50B). The intensity of the fluorescence signals was then normalized and the\% of doxorubicin release was calculated using Eq. (3);

$\%$ Release $=[I(s)-I(0)] /[I(t)-I(0)] * 100$

Where, I(s) is the fluorescence intensity of individual samples at different time points, $I(0)$ is the background fluorescence intensity of liposome samples after purification and $\mathrm{I}(\mathrm{t})$ is the fluorescence intensity of liposomes suspension after the addition of $2 \mu \mathrm{l}$ of $10 \%$ Triton X-100 in HBS ( $\mathrm{pH} 7.4)$.

\section{Results}

\subsection{Engineering thermosensitive liposome-NPs hybrids}

Thermosensitive liposome-NP hybrids were engineered using the reverse phase evaporation method (Szoka and Papahadjopoulos, 1978) based on the hypothesis that significantly 
higher NP incorporation within the lipid bilayer would be afforded. To test this, two alternative reverse phase evaporation-based protocols were compared, namely, the original reverse phase evaporation [RP] and the modified lipid film hydration-reverse phase evaporation [F-RP], aiming to maintain the thermal sensitivity of the lysolipid-containing LTSL (Fig. 1A).

First, plain LTSL liposomes were prepared by both RP and F-RP that did not show a significant difference in mean vesicle diameter and surface properties (Fig. 1B). However, fluorescence anisotropy studies and a doxorubicin release experiment indicated significant differences between the two protocols. Fluorescence anisotropy studies were performed using two different types of membrane-bound probes (DPH and ANS) to allow monitoring of the molecular interactions at the alkyl chain and head group regions of the vesicle bilayer, respectively. Higher anisotropy values were observed from LTSL liposomes prepared with the RP protocol, indicating an increase in bilayer rigidity compared to the F-RP protocol (Fig. $1 \mathrm{Ci}$, ii). This was also associated with a reduction in the temperature-responsiveness of the LTSL-RP illustrated by the limited doxorubicin release after $60 \mathrm{~min}$ of exposure to mild HT $\left(42^{\circ} \mathrm{C}\right)$. In comparison, LTSL liposomes prepared with F-RP protocol preserved their thermoresponsiveness evidenced by complete drug content release (>90\% release) at $42{ }^{\circ} \mathrm{C}$ (Fig. 1Civ).

Based on the above and in order to preserve the thermal sensitivity of LTSL liposomes, the F-RP protocol was chosen for the engineering of the LTSL-NP hybrid vesicles.

Thermosensitive liposome-NP hybrids were designed to incorporate the metallic NPs within the phospholipids bilayer. Three different types of metallic NPs were studied: AuNPs, AgNPs and SPIO NPs. In order to ensure that the NPs were embedded in the lipid bilayer, hydrophobic NPs with a size that is comparable to, or smaller than the thickness of a lipid membrane ( $<5 \mathrm{~nm}$ ) were selected (Fig. 2A) (Al-Jamal et al., 2008). 


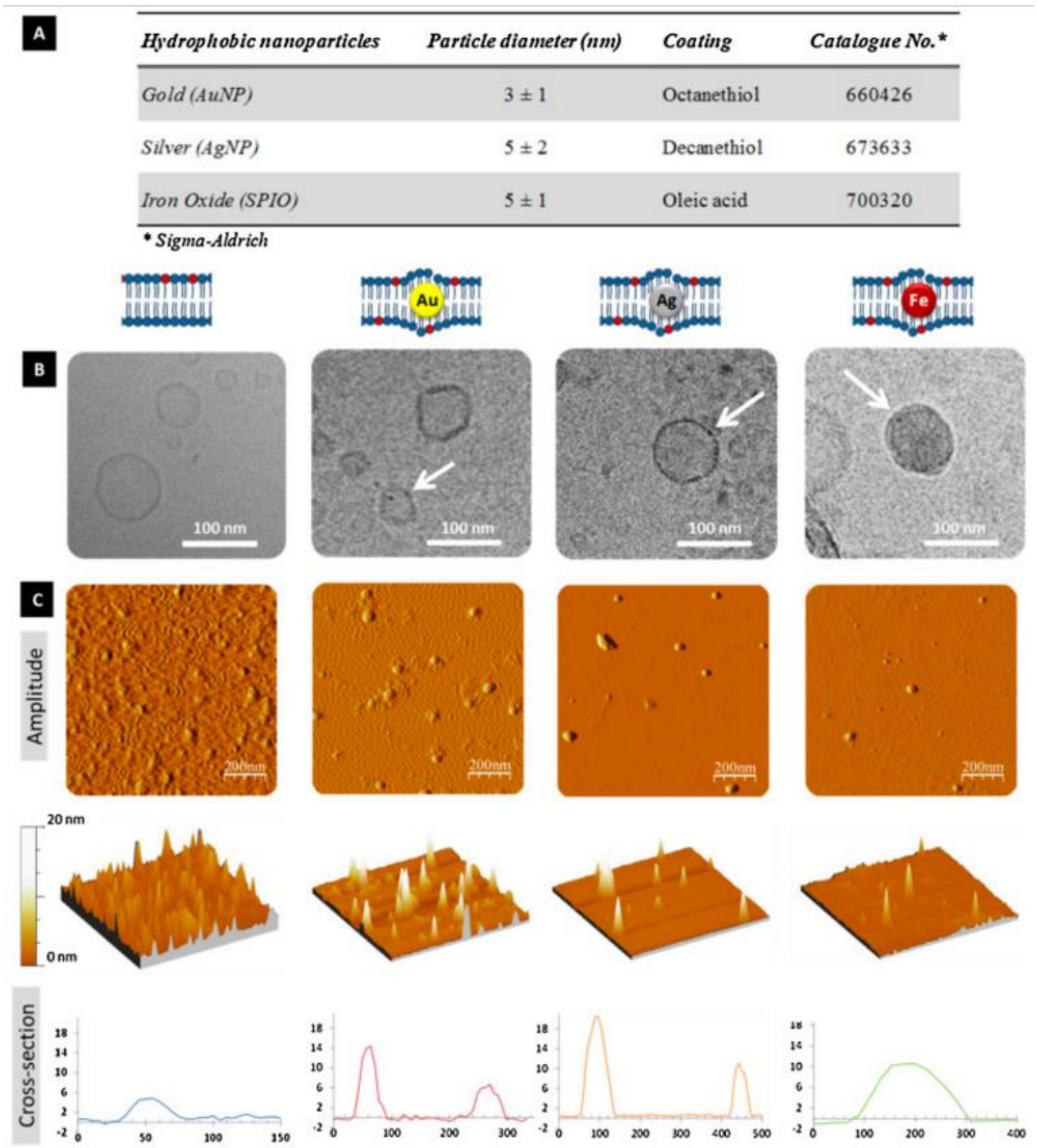

Fig. 2.

(A) Hydrophobic NPs characteristics. (B) Cryo-EM micrographs showing the morphology and incorporation of metallic NPs in the lipid bilayer of the liposomes as indicated by the arrows, and (C) AFM (amplitude and 3D images and cross-section analysis) for $5 \mathrm{mM}$ LTSL without and with $10 \mu \mathrm{g} / \mathrm{ml}$ of AuNPs, AgNPs and SPIO NPs.

\subsection{Cryo-EM and AFM imaging of thermosensitive liposome-NP hybrids}

The incorporation of all three types of metallic NP into LTSL vesicle bilayers by the F-RP method was performed and the morphological characterization of the ensuing hybrid systems was studied by cryo-EM and AFM. Although the exact quantification of metallic NPs embedded in the liposomes was not quantified, we have studied the interaction between metallic NP within the LTSL vesicles using cryo-EM and AFM imaging. LTSL-NPs hybrids imaged by cryo-EM, indicated that the incorporation of the NP into the lipid bilayer was taking place (Fig. 2B, see arrows). Surface topology of LTSL-NP hybrids was also studied with 
AFM. Interestingly, AFM cross-section analysis (Fig. 2C) suggested that metallic NP incorporation into LTSL increased the height (i.e. thickness) of the lipid bilayer from 6 to 8 $\mathrm{nm}$ to almost $18-20 \mathrm{~nm}$. This was in agreement with previously reported studies from other liposome hybrid systems (Tian et al., 2011). Overall, the microscopy evidence obtained by cryo-EM and AFM corroborated to suggest that the metallic NPs were localized within the lipid bilayers.

\subsection{The effect of NP concentration on hybrid system properties}

To study the effect of the incorporated NP concentration on the properties of LTSL hybrid systems, AuNP were chosen as it is considered the most popular NP for efficient light induced heating and attracted great attention in the past for their multifunctional character. Two different concentrations of AuNPs were selected for these studies; 5 and $10 \mu \mathrm{g} / \mathrm{ml}$. Incorporation of AuNP at both concentrations resulted in hybrid systems less than $100 \mathrm{~nm}$ in mean diameter, low polydispersity index (PDI) and a slightly negative surface charge (Fig. 3A). Fluorescence anisotropy studies showed higher $r$ values for the LTSL-AuNP hybrids (compared to LTSL alone) indicating an increase in bilayer rigidity by the incorporation of AuNPs (Fig. 3Bi, ii). LTSL-AuNP hybrids were successfully loaded with the anticancer drug doxorubicin and maintained good stability at body temperature as evidenced by drug retention at $37^{\circ} \mathrm{C}$ (Fig. 3Ciii). Interestingly, a concentration-dependent modulation of doxorubicin release at mild $\mathrm{HT}\left(42^{\circ} \mathrm{C}\right)$ was observed by the incorporation of AuNP within the LTSL bilayers (Fig. 3Civ), that could be expected as a result of the anisotropy-determined increase in bilayer rigidity. 


\begin{tabular}{|c|c|c|c|c|}
\hline System & Hydrodinamic diameter ${ }^{a}(\mathrm{~nm})$ & PDI" & $\zeta$-Potential ${ }^{a}(m V)$ & $E E^{b} \%$ \\
\hline LTSL-5 $\mu g / m L$ AuNP & $81.1 \pm 1.7$ & $0.277 \pm 0.022$ & $-11.6 \pm 1.9$ & 81 \\
\hline 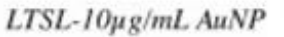 & $85.7 \pm 0.4$ & $0.272 \pm 0.006$ & $-7.1 \pm 1.1$ & 82 \\
\hline
\end{tabular}

"Concentration of LTSL (low themosensitive lposones) [F-RP] of $5 \mathrm{~mm}$.

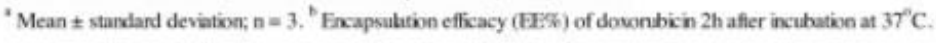
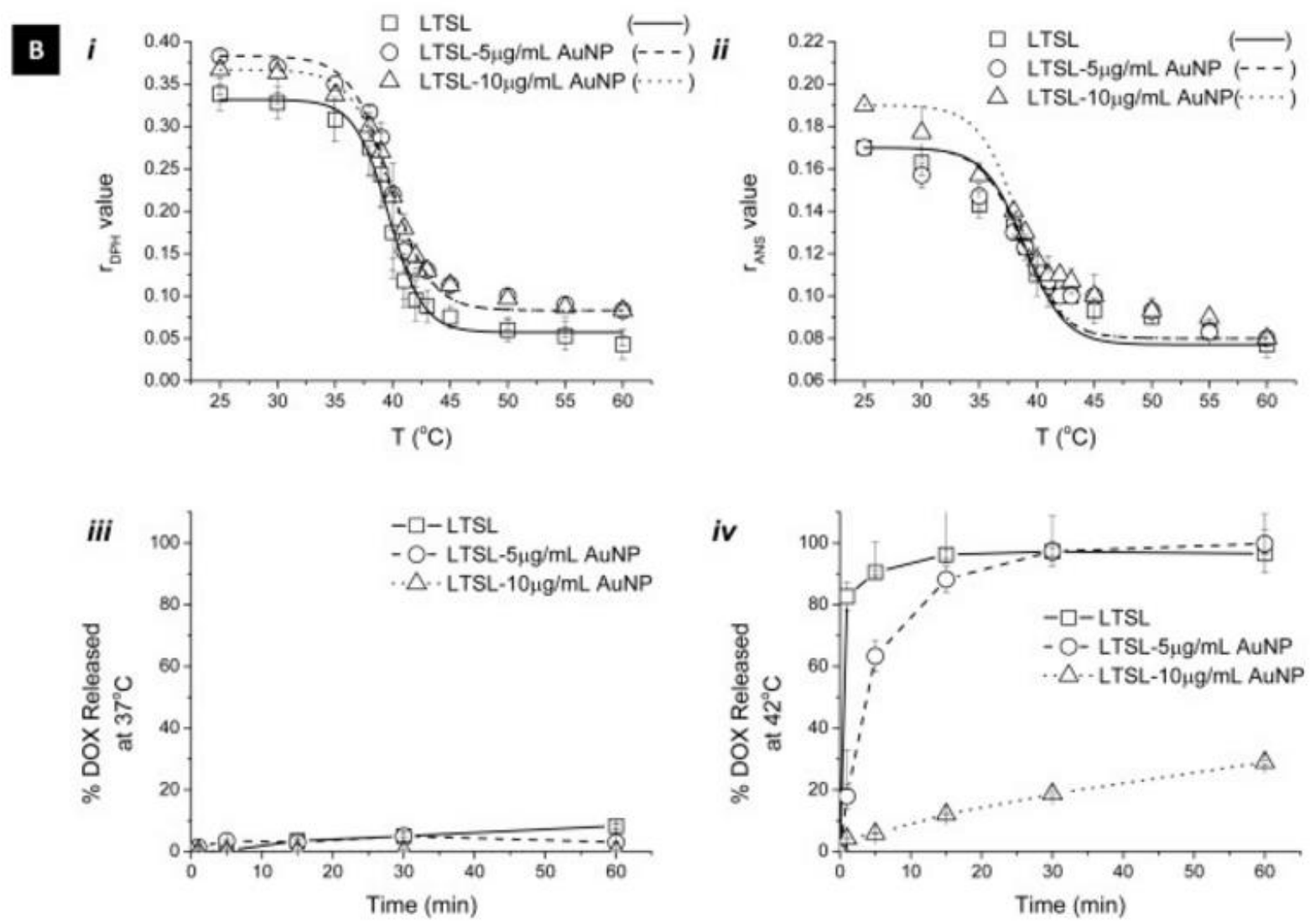

Fig. 3.

(A) Colloidal stability summary and doxorubicin encapsulation efficiency (EE\%) of LTSL and LTSL-AuNP hybrids. Data obtained by Nanosizer ZS and fluorescence spectrophotometer. (B) Fluorescence anisotropy of (i) DPH and (ii) ANS and\% doxorubicin released at (iii) $37^{\circ} \mathrm{C}$ and (iv) $42^{\circ} \mathrm{C}$ for $5 \mathrm{mM} \mathrm{LTSL}$ (squares and solid lines) and LTSL embedded with 5 (circles and dashed lines) and 10 (up-triangles and dotted lines) $\mu \mathrm{g} / \mathrm{ml}$ of AuNPs.

\subsection{The effect of metal NP type on hybrid system properties}

The effect of NP type on the hybrid vesicle characteristics and temperature-triggered release properties was investigated next. Three different types of metallic NPs were compared by allowing their incorporation into LTSL lipid bilayers at $10 \mu \mathrm{g} / \mathrm{ml}$ using the F-RP protocol. Physicochemical characterization of LTSL-AuNP/AgNP/SPIO hybrid systems revealed that all LTSL-NP hybrids resulted in mean vesicle diameter (and PDI) comparable to LTSL alone (Fig. 4A). It is also worth noting that the bilayer Tt after incorporation of the three different metallic NPs remained consistent with that of the LTSL alone (between 40.5$41^{\circ} \mathrm{C}$ ). The three types of LTSL-NP hybrid vesicles were found to be more surface charge neutral than their plain LTSL liposome counterpart. Interestingly, all LTSL-NP hybrid systems were able to be remote-loaded with doxorubicin at a higher encapsulation efficiency compared to LTSL liposomes prepared by F-RP. The encapsulation efficiency of all tested 
were almost comparable to what observed from LTSL-RP. The presence of NPs in the lipid bilayer of LTSL liposomes prepared by F-RP method may offer better preservation of the $\mathrm{pH}$ gradient required for doxorubicin loading process. This could be due to the increase in bilayer rigidity in the presence of NPs compared to plain LTSL. As observed from florescence anisotropy studies. This again indicated that the incorporation of metallic NPs into LTSL lipid bilayers resulted in a higher bilayer rigidity revealed by an increase in $r$ values (Fig. 4Bi, ii), which was almost comparable to $r$ values observed before from LTSL-RP (Fig. 1Ci). This increase in bilayer rigidity was observed both in the core and head group region of the phospholipid membrane irrespective to the type of the NPs incorporated, even though it did not lead to a change in the overall Tt of the bilayer as noted above. In terms of doxorubicin release profile, similar to LTSL-AuNP hybrids described above, LTSL-AgNP and LTSL-SPIO hybrids maintained good drug retention at body temperature ( Fig. 4Biii), while offering controlled drug release for 60 min under mild HT conditions (Fig. 4Biv). All three types of LTSL-NP hybrids released doxorubicin at significantly lower rates compared to the LTSL liposomes. Overall, no significant differences were observed in both physicochemical and drug release characteristics among the three different types of LTSL-NP hybrids, in contrast to the sharp differences in comparison to LTSL liposomes alone. 


\begin{tabular}{|c|c|c|c|c|c|}
\hline System ${ }^{*}$ & Hydrodinamic diameter ${ }^{*}(\mathrm{~mm})$ & $P D I^{\alpha}$ & c-Potential ${ }^{a}(m V)$ & $\left.T,{ }^{\circ} \mathrm{C}\right)$ & $E E^{b} \%$ \\
\hline LTSL & $73.0 \pm 0.6$ & $0.304 \pm 0.032$ & $-18.8 \pm 0.1$ & $40.98 \pm 0.03$ & 66 \\
\hline LTSL-AuNP & $85.7 \pm 0.4$ & $0.272 \pm 0.006$ & $-7.1 \pm 1.1$ & $40.49 \pm 0.08$ & 82 \\
\hline LTSL-AgNP & $89.6 \pm 4.6$ & $0.344 \pm 0.007$ & $-6.6 \pm 0.5$ & $40.67 \pm 0.12$ & 90 \\
\hline LTSL-SPIO & $74.8 \pm 0.3$ & $0.245 \pm 0.009$ & $-7.1 \pm 0.1$ & $41.04 \pm 0.09$ & 83 \\
\hline
\end{tabular}

Concentration of LTSL. (bw thenwosenstive fiposones) [F-RP] of $5 \mathrm{mM}$. Coscentration of nanoparticles 10ugini.

"Mean \pm standard deviation, $\mathrm{n}=3$, "Encapsubtion efficacy (EE) of downbicin $2 \mathrm{~h}$ after inculation at $37 \mathrm{C}$.

B $\boldsymbol{i}$
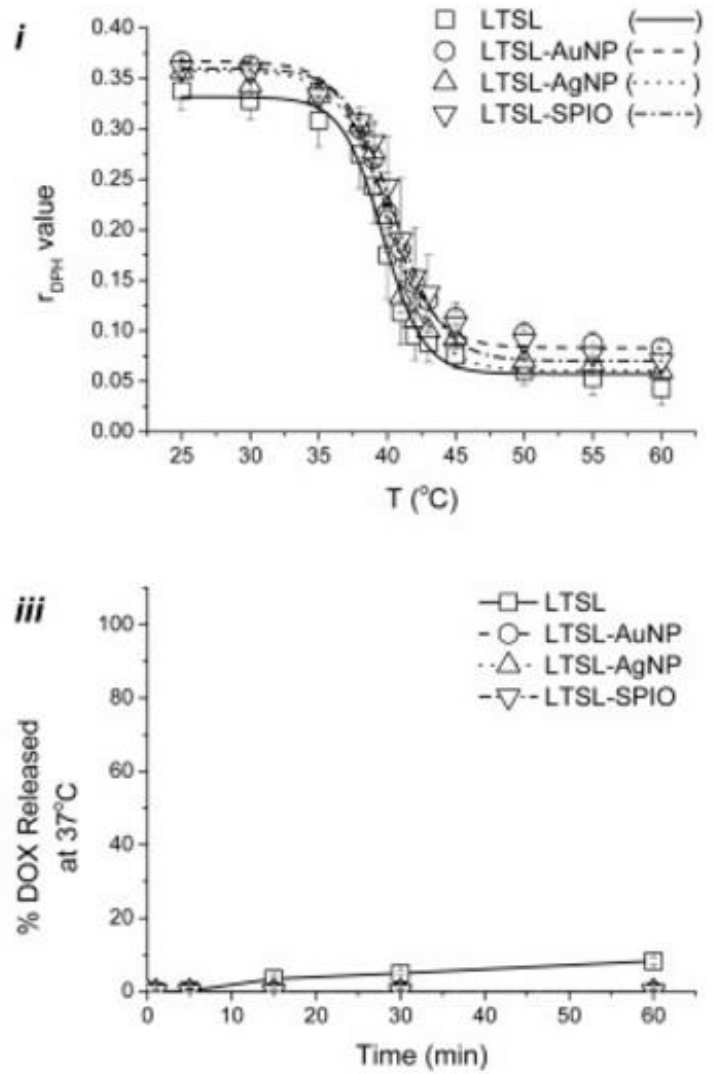

ii

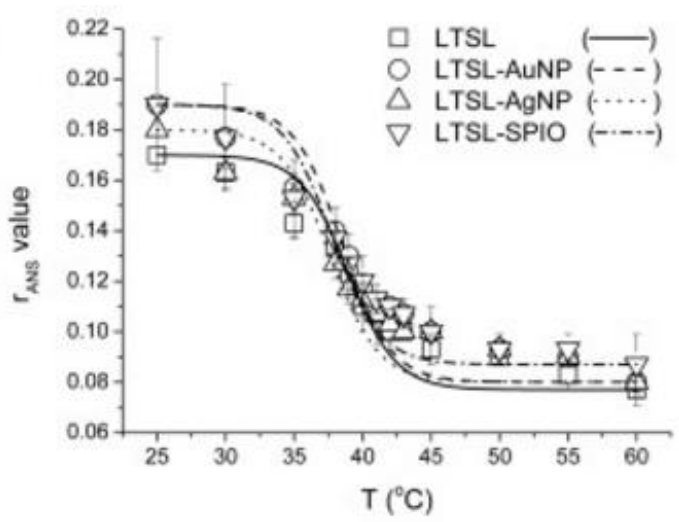

iv

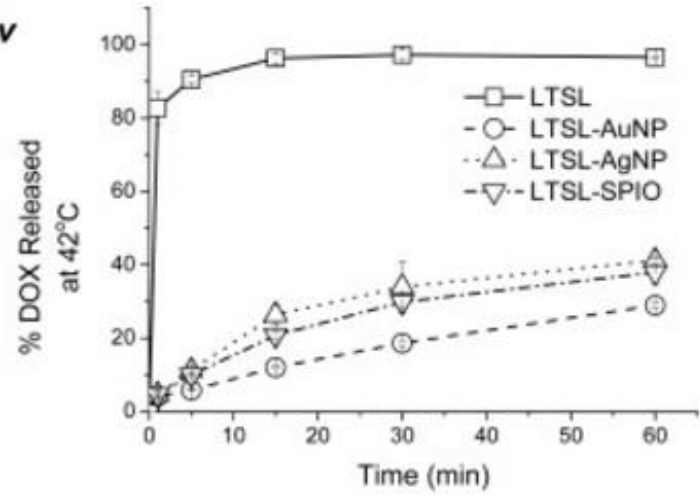

Fig. 4.

(A) Colloidal stability summary and doxorubicin encapsulation efficiency (EE\%) of LTSL and LTSLNP hybrids. Data obtained by Nanosizer ZS and fluorescence spectrophotometer. (B) Fluorescence anisotropy of (i) DPH and (ii) ANS and $1 \mathrm{~h} \%$ doxorubicin released at (iii) $37^{\circ} \mathrm{C}$ and (iv) $42^{\circ} \mathrm{C}$ for $5 \mathrm{mM} \mathrm{LTSL}$ (squares and solid lines) and LTSL embedded with $10 \mu \mathrm{g} / \mathrm{ml}$ of AuNPs (circles), AgNPs (up-triangles), and SPIO NPs (down-triangles).

\section{Discussion}

Even though liposomes and metallic NPs are independently already in clinical practice, the combination of liposome-NP hybrids is still an exploratory approach to design a new generation of multifunctional delivery systems (Preiss and Bothun, 2011). The aim of such hybrid systems is to combine the capabilities of both liposomes and NPs and therefore provide an advanced platform for theranostic applications. Thermosensitive liposome-NP hybrid systems are interesting examples of such design strategies as they aim to take advantage of the thermal properties of NPs in order to achieve remote drug release (Pradhan et al., 2010 and Volodkin et al., 2009). Several examples of thermosensitive liposome-NP hybrids have been proposed recently by incorporating NPs either in the 
aqueous core, lipid membrane or by surface adsorption. From these three different designs, membrane-embedded and surface-adsorbed NPs showed the highest content release. This effect was studied in detail by Passonen et al. by incorporating 2-4 nm AuNPs into DPPC:DSPC TSL to report the percentage of calcein release after 5-10 min of continuous exposure to UV light at $250 \mathrm{~nm}$ (Paasonen et al., 2007). Maximum release was achieved from membrane embedded Au-C6SH NPs and surface adsorbed DPPE-NanoAu ${ }^{\circledR}$ NPs. Similarly, the extent of carboxyfluorescein dye release from DPPC liposomes decorated with hollow Au nanoshells (Wu, 2008) and DPPC:DPTAP:CHOL-AuNPs complexes (Volodkin et al., 2009) was highly dependent on the proximity of the NPs to the lipid bilayer. The reason behind that was possibly due to a more efficient and localized direct heat transport from the NPs to the liposomal bilayer compared to the encapsulated AuNPs (Paasonen et al., 2007). These results also suggested that the mechanism behind the observed triggered release could be due to the formation of transient pores in the bilayer or due to other forms of mechanical disruption of the lipid bilayer by the incorporated NPs. Fluorescence anisotropy has also been widely used to study the effect of NPs incorporation on lipid membrane packing and fluidity, showing that this is largely dependent on the concentration, size, position of NPs incorporated and the temperature tested (Park et al., 2005, Park et al., 2006 and Park et al., 2009).

In agreement with our findings, incorporation of small hydrophobic NPs $(<5 \mathrm{~nm})$ decreased the lipid membrane fluidity (membrane rigidifying effect) particularly at temperatures below the phase transition temperature of the lipid membrane (Park et al., 2005, Park et al., 2006 and Park et al., 2009). Membrane rigidity was augmented with increasing the NP concentration. The most likely explanation for such effects is the small size of NPs (smaller than the thickness of bilayer) that allows for complete NP incorporation within the lipid bilayer (Park et al., 2005, Park et al., 2006 and Park et al., 2009). Increased bilayer rigidity can also explain the different rates of drug release observed from the three types of liposome-NP hybrids we studied here. This observation also agreed with a previous report by Chen et al. who showed that the release of an encapsulated dye molecule $(5,6$ Carboxyfluorescein) from DPPC liposomes was reduced by increasing the amount of SPIO NPs embedded within the lipid membrane (Chen et al., 2010). Interestingly, that study also showed that content leakage was enhanced when triggered by an external magnetic field, further suggesting the critical role played by the incorporated NPs (Chen et al., 2010). The opposite effect on lipid membrane rigidity was observed when the incorporated NPs were near or just exceeding the thickness of the lipid membrane. Increases in DPPC membrane fluidity and a decreased vesicle phase transition temperature were reported by the incorporation of AgNPs larger than $5 \mathrm{~nm}$ (Bothun, 2008). The distortion of the lipid bilayer to accommodate the NPs was suggested as responsible for the decrease in lipid ordering and increased membrane fluidity (Bothun, 2008). No significant effect on bilayer fluidity was reported from NPs encapsulated in the aqueous core (Bothun, 2008 and Preiss and Bothun, 2011).

Despite previous reports of interesting new designs of thermosensitive liposome-NP hybrids, only a few studies have investigated the therapeutic relevance of these systems by the encapsulation of clinically-used drug molecules. These examples involved the coencapsulation of both therapeutic drug molecules, such as doxorubicin (Pradhan et al., 2010) or methotrexate (Zhu et al., 2009), and metallic NPs in the liposomal aqueous 
compartment and showed interesting effects in response to external stimuli (Pradhan et al., 2010 and Zhu et al., 2009).

The capability of combining therapeutic and imaging functionalities in a single liposome-NP hybrid system can provide an attractive theranostic approach for many diseases in which both diagnosis and therapy need to be combined. Such hybrid systems can be imaged to offer non-invasive assessment blood kinetics and tissue distribution, targeted at specific cells and tissue, as well as offer controlled drug release at the target site on external stimulation. Consequently, this would provide the potential to have better prediction of the therapeutic activity and open new insights into more personalized medications. In the present study, we reported thermosensitive liposome-NP hybrid systems by incorporating different types of metallic NPs in the bilayer of clinically-developed LTSL formulations. Altering the concentration and the type of the NPs we can effectively control drug release from LTSL liposomes without interference with drug loading and drug retention. LTSL-NP hybrid systems present a potential multifunctional vector of great modular capacity, whose properties can be modulated by the type of NPs incorporated within the lipid bilayer.

More research is warranted to optimize the choice of TSL formulation and biocompatibility of the liposome-NP hybrids. In addition, the structural integrity of the liposome-NP hybrids in vivo needs to be further investigated to address the possibility of NP release from liposomes during blood circulation and the consequences this could have on drug retention, drug pharmacology and long term safety profile. Furthermore, the therapeutic and imaging potential of liposome-NP hybrids needs to be further explored using clinically relevant noninvasive external triggering techniques.

\section{Conclusions}

In conclusion, we present the engineering of doxorubicin-loaded thermosensitive liposomeNPs hybrids as potential theranostic platforms. The use of metallic NPs with mean diameters smaller than the thickness of lipid membrane ensured successful incorporation within the vesicle lipid bilayer as evidenced with cryo-EM and AFM studies. As a result of that an increase in bilayer rigidity and tunable doxorubicin release profile from the hybrid systems was obtained after heating. Our data suggested that this approach can provide an alternative strategy to design stable liposome-NP hybrids with additional functionalities that are contributed by the type of metallic NPs incorporated. We suggest that LTSL-NP hybrids may offer multifunctional delivery systems with the potential to achieve simultaneous tracking and triggered-release.

\section{Competing financial interests}

The authors declare no competing financial interests.

\section{Author contributions}

Z.A., N.L. and K.K. wrote the manuscript text. N.L., K.C.M. and K.K. prepared the figures. K.C.M. and N.L. performed the experimental work and data analysis. Z.A., N.L., W.A., K.K. planned experimental work and provided technical training. Z.A, N.L, K.C.M. and K.K. reviewed the manuscript.

\section{Acknowledgments}


This work was partially supported by the Andalusian Initiative for Advanced Therapies promoted by the Regional Government of Andalusia, Spain (to N.L.), European Commission FP7 Program SONODRUGS (NMP4-LA-2008-213706) and by UCL School of Pharmacy. We would like to acknowledge $\operatorname{Dr}$ Marc C. A. Stuart from the Electron Microscopy Unit at the Groningen Institute of Biomolecular Sciences and Biotechnology for the Cryo-EM imaging. The authors acknowledge Lipoid Co. (Germany) for the lipid sample gifts.

\section{References}

Al-Ahmady and Kostarelos, 2016

Z. Al-Ahmady, K. Kostarelos

Chemical components for the design of temperature-responsive vesicles as cancer therapeutics

Chem. Rev., 116 (2016), pp. 3883-3918

Al-Ahmady et al., 2012

Z.S. Al-Ahmady, W.T. Al-Jamal, J.V. Bossche, T.T. Bui, A.F. Drake, A.J. Mason, K. Kostarelos Lipid-peptide vesicle nanoscale hybrids for triggered drug release by mild hyperthermia in vitro and in vivo

ACS Nano, 6 (2012), pp. 9335-9346

Al-Ahmady et al., 2014

Z.S. Al-Ahmady, O. Chaloin, K. Kostarelos

Monoclonal antibody-targeted, temperature-sensitive liposomes: in vivo tumor chemotherapeutics in combination with mild hyperthermia

J. Controlled Release, 196 (2014), pp. 332-343

Al-Ahmady et al., 2015

Z.S. Al-Ahmady, C.L. Scudamore, K. Kostarelos

Triggered doxorubicin release in solid tumors from thermosensitive liposome-peptide hybrids: critical parameters and therapeutic efficacy

Int. J. Cancer, 137 (2015), pp. 731-743

Al-Jamal et al., 2008

W.T. Al-Jamal, K.T. Al-Jamal, B. Tian, L. Lacerda, P.H. Bornans, P.M. Frederik, K. Kostarelos Lipid-quanturn dot bilayer vesicles enhance tumor cell uptake and retention in vitro and in vivo

ACS Nano, 2 (2008), pp. 408-418

Alkilany et al., 2012

A.M. Alkilany, L.B. Thompson, S.P. Boulos, P.N. Sisco, C.J. Murphy

Gold nanorods: their potential for photothermal therapeutics and drug delivery, tempered by the complexity of their biological interactions

Adv. Drug Delivery Rev., 64 (2012), pp. 190-199

Allen and Cullis, 2013

T.M. Allen, P.R. Cullis 
Liposomal drug delivery systems: from concept to clinical applications

Adv. Drug Delivery Rev., 65 (2013), pp. 36-48

Barenholz, 2012

Y. Barenholz

Doxil $^{\circledR}$-the first FDA-approved nano-drug: lessons learned

J. Controlled Release, 160 (2012), pp. 117-134

Bealle et al., 2012

G. Bealle, R. Di Corato, J. Kolosnjaj-Tabi, V. Dupuis, O. Clement, F. Gazeau, C. Wilhelm, C. Menager

Ultra magnetic liposomes for MR imaging, targeting, and hyperthermia

Langmuir, 28 (2012), pp. 11834-11842

Bothun, 2008

G.D. Bothun

Hydrophobic silver nanoparticles trapped in lipid bilayers: size distribution, bilayer phase behavior, and optical properties

J. Nanobiotechnol., 6 (2008), p. 13

Chanda et al., 2010

N. Chanda, P. Kan, L.D. Watkinson, R. Shukla, A. Zambre, T.L. Carmack, H. Engelbrecht, J.R. Lever, K. Katti, G.M. Fent, S.W. Casteel, C.J. Smith, W.H. Miller, S. Jurisson, E. Boote, J.D. Robertson, C. Cutler, M. Dobrovolskaia, R. Kannan, K.V. Katti

Radioactive gold nanoparticles in cancer therapy: therapeutic efficacy studies of GA198AuNP nanoconstruct in prostate tumor-bearing mice

Nanomed.: Nanotechnol. Biol. Med., 6 (2010), pp. 201-209

Chen et al., 2010

Y. Chen, A. Bose, G.D. Bothun

Controlled release from bilayer-decorated magnetoliposomes via electromagnetic heating ACS Nano, 4 (2010), pp. 3215-3221

Chithrani et al., 2010

D.B. Chithrani, M. Dunne, J. Stewart, C. Allen, D.A. Jaffray

Cellular uptake and transport of gold nanoparticles incorporated in a liposomal carrier Nanomedicine, 6 (2010), pp. 161-169

Clesion.com, 2016

Clesion.com, 2016. Celsion Presents Data on ThermoDox ${ }^{\circledR}$ plus Optimized RFA in Intermediate HCC.

Gabizon and Martin, 1997

A. Gabizon, F. Martin

Polyethylene glycol-coated (pegylated) liposomal doxorubicin: rationale for use in solid tumours

Drugs, 4 (1997), pp. 15-21 
Gabizon et al., 1994

A. Gabizon, R. Catane, B. Uziely, B. Kaufman, T. Safra, R. Cohen, F. Martin, A. Huang, Y. Barenholz

Prolonged circulation time and enhanced accumulation in malignant exudates of doxorubicin encapsulated in polyethylene-glycol coated liposomes

Cancer Res., 54 (1994), pp. 987-992

Grzelczak and Liz-Marzan, 2013

M. Grzelczak, L.M. Liz-Marzan

Colloidal nanoplasmonics: from building blocks to sensing devices

Langmuir, 29 (2013), pp. 4652-4663

Hormeno et al., 2014

S. Hormeno, P. Gregorio-Godoy, J. Perez-Juste, L.M. Liz-Marzan, B.H. Juarez, J.R. AriasGonzalez

Laser heating tunability by off-resonant irradiation of gold nanoparticles

Small, 10 (2014), pp. 376-384

Jain et al., 2003

S. Jain, V. Mishra, P. Singh, P.K. Dubey, D.K. Saraf, S.P. Vyas

RGD-anchored magnetic liposomes for monocytes/neutrophils-mediated brain targeting Int. J. Pharm., 261 (2003), pp. 43-55

Johannsen et al., 2010

M. Johannsen, B. Thiesen, P. Wust, A. Jordan

Magnetic nanoparticle hyperthermia for prostate cancer

Int. J. Hyperthermia, 26 (2010), pp. 790-795

Jordan et al., 2006

A. Jordan, R. Scholz, K. Maier-Hauff, F.K. van Landeghem, N. Waldoefner, U. Teichgraeber, J. Pinkernelle, H. Bruhn, F. Neumann, B. Thiesen, A. von Deimling, R. Felix

The effect of thermotherapy using magnetic nanoparticles on rat malignant glioma

J. Neurooncol., 78 (2006), pp. 7-14

Kojima et al., 2008

C. Kojima, Y. Hirano, E. Yuba, A. Harada, K. Kono

Preparation and characterization of complexes of liposomes with gold nanoparticles

Colloids Surf. B, 66 (2008), pp. 246-252

Koudelka and Turánek, 2012

Š. Koudelka, J. Turánek

Liposomal paclitaxel formulations

J. Controlled Release, 163 (2012), pp. 322-334

Landon et al., 2011

C.D. Landon, J.Y. Park, D. Needham, M.W. Dewhirst 
Nanoscale drug delivery and hyperthermia: the materials design and preclinical and clinical testing of low temperature-Sensitive liposomes used in combination with mild hyperthermia in the treatment of local cancer

Open Nanomed. J., 3 (2011), pp. 38-64

Li et al., 1998

X. Li, D.J. Hirsh, D. Cabral-Lilly, A. Zirkel, S.M. Gruner, A.S. Janoff, W.R. Perkins

Doxorubicin physical state in solution and inside liposomes loaded via a $\mathrm{pH}$ gradient

Biochim. Biophys. Acta Biomembr., 1415 (1998), pp. 23-40

Liu et al., 2012

M. Liu, L. Gan, L. Chen, D. Zhu, Z. Xu, Z. Hao, L. Chen

A novel liposome-encapsulated hemoglobin/silica nanoparticle as an oxygen carrier Int. J. Pharm., 427 (2012), pp. 354-357

Lozano et al., 2012

N. Lozano, W.T. Al-Jamal, A. Taruttis, N. Beziere, N.C. Burton, J. Van den Bossche, M. Mazza, E. Herzog, V. Ntziachristos, K. Kostarelos

Liposome-gold nanorod hybrids for high-resolution visualization deep in tissues

J. Am. Chem. Soc., 134 (2012), pp. 13256-13258

Lozano et al., 2015

N. Lozano, Z.S. Al-Ahmady, N.S. Beziere, V. Ntziachristos, K. Kostarelos

Monoclonal antibody-targeted PEGylated liposome-ICG encapsulating doxorubicin as a potential theranostic agent

Int. J. Pharm., 482 (2015), pp. 2-10

Maestro et al., 2014

L.M. Maestro, P. Haro-Gonzalez, A. Sanchez-Iglesias, L.M. Liz-Marzan, J. Garcia Sole, D. Jaque Quantum dot thermometry evaluation of geometry dependent heating efficiency in gold nanoparticles

Langmuir, 30 (2014), pp. 1650-1658

Maier-Hauff et al., 2007

K. Maier-Hauff, R. Rothe, R. Scholz, U. Gneveckow, P. Wust, B. Thiesen, A. Feussner, A. von Deimling, N. Waldoefner, R. Felix, A. Jordan

Intracranial thermotherapy using magnetic nanoparticles combined with external beam radiotherapy: results of a feasibility study on patients with glioblastoma multiforme

J. Neurooncol., 81 (2007), pp. 53-60

Paasonen et al., 2007

L. Paasonen, T. Laaksonen, C. Johans, M. Yliperttula, K. Kontturi, A. Urtti

Gold nanoparticles enable selective light-induced contents release from liposomes

J. Controlled Release, 122 (2007), pp. 86-93

Paasonen et al., 2010 
L. Paasonen, T. Sipila, A. Subrizi, P. Laurinmaki, S.J. Butcher, M. Rappolt, A. Yaghmur, A. Urtti, M. Yliperttula

Gold-embedded photosensitive liposomes for drug delivery: triggering mechanism and intracellular release

J. Controlled Release, 147 (2010), pp. 136-143

Park et al., 2005

S.-H. Park, S.-G. Oh, J.-Y. Mun, S.-S. Han

Effects of silver nanoparticles on the fluidity of bilayer in phospholipid liposome

Colloids Surf. B, 44 (2005), pp. 117-122

Park et al., 2006

S.-H. Park, S.-G. Oh, J.-Y. Mun, S.-S. Han

Loading of gold nanoparticles inside the DPPC bilayers of liposome and their effects on membrane fluidities

Colloids Surf. B, 48 (2006), pp. 112-118

Park et al., 2009

S.-H. Park, S.-G. Oh, K.-D. Suh, S.-H. Han, D.J. Chung, J.-Y. Mun, S.-S. Han, J.-W. Kim Control over micro-fluidity of liposomal membranes by hybridizing metal nanoparticles

Colloids Surf. B, 70 (2009), pp. 108-113

Pornpattananangkul et al., 2010

D. Pornpattananangkul, S. Olson, S. Aryal, M. Sartor, C.M. Huang, K. Vecchio, L. Zhang Stimuli-responsive liposome fusion mediated by gold nanoparticles

ACS Nano, 4 (2010), pp. 1935-1942

Pradhan et al., 2010

P. Pradhan, J. Giri, F. Rieken, C. Koch, O. Mykhaylyk, M. Doblinger, R. Banerjee, D. Bahadur,

C. Plank

Targeted temperature sensitive magnetic liposomes for thermo-chemotherapy

J. Controlled Release, 142 (2010), pp. 108-121

Preiss and Bothun, 2011

M.R. Preiss, G.D. Bothun

Stimuli-responsive liposome-nanoparticle assemblies

Expert Opin. Drug Delivery, 8 (2011), pp. 1025-1040

Sawant and Torchilin, 2012

R.R. Sawant, V.P. Torchilin

Challenges in development of targeted liposomal therapeutics

AAPS J., 14 (2012), pp. 303-315

Soga et al., 2010

K. Soga, K. Tokuzen, K. Tsuji, T. Yamano, H. Hyodo, H. Kishimoto

NIR bioimaging: development of liposome-Encapsulated, rare-earth-doped Y2O3

nanoparticles as fluorescent probes 
Eur. J. Inorg. Chem. (2010), pp. 2673-2677

Szoka and Papahadjopoulos, 1978

F. Szoka Jr., D. Papahadjopoulos

Procedure for preparation of liposomes with large internal aqueous space and high capture by reverse-phase evaporation

Proc. Natl. Acad. Sci. U. S. A., 75 (1978), pp. 4194-4198

Tai et al., 2009

L.-A. Tai, P.-J. Tsai, Y.-C. Wang, Y.-J. Wang, L.-W. Lo, C.-S. Yang

Thermosensitive liposomes entrapping iron oxide nanoparticles for controllable drug release Nanotechnology, 20 (2009), p. 135101

Tian et al., 2011

B. Tian, W.T. Al-Jamal, K.T. Al-Jamal, K. Kostarelos

Doxorubicin-loaded lipid-quantum dot hybrids: surface topography and release properties Int. J. Pharm., 416 (2011), pp. 443-447

Visaria et al., 2006

Visaria, R.K. 2006Visaria, R.J. Griffin, B.W. Williams, E.S. Ebbini, G.F. Paciotti, C.W. Song, J.C. Bischof, et al.

Enhancement of tumor thermal therapy using gold nanoparticle-assisted tumor necrosis factor- $\alpha$ delivery

Mol. Cancer Ther., 5 (2006), pp. 1014-1020

Volodkin et al., 2009

D.V. Volodkin, A.G. Skirtach, H. Mohwald

Near-IR remote release from assemblies of liposomes and nanoparticles

Angew. Chem. Int. Ed. Engl., 48 (2009), pp. 1807-1809

Wong and Liu, 2010

K.K.Y. Wong, X. Liu

Silver nanoparticles-the real silver bullet in clinical medicine?

MedChemComm, 1 (2010), pp. 125-131

Wu, 2008

$\mathrm{G}$. Wu

Remotely triggered liposome release by near-infrared light absorption via hollow gold nanoshells

J. Am. Chem. Soc., 130 (2008), pp. 8175-8177

Zhu et al., 2009

L. Zhu, Z.L. Huo, L.L. Wang, X. Tong, Y. Xiao, K.Y. Ni

Targeted delivery of methotrexate to skeletal muscular tissue by thermosensitive magnetoliposomes

Int. J. Pharm., 370 (2009), pp. 136-143 
Corresponding author at: Nanomedicine Lab, Faculty of Biology, Medicine \& Health, University of Manchester, AV Hill Building, Oxford Road, Manchester M13 9PT, United Kingdom. 